\title{
Stratification for Confounding - Part 1: The Mantel-Haenszel Formula
}

\author{
Giovanni Tripepi $^{\mathrm{a}}$ Kitty J. Jager ${ }^{\mathrm{b}}$ Friedo W. Dekker ${ }^{\mathrm{b}, \mathrm{c}}$ Carmine Zoccali ${ }^{\mathrm{a}}$ \\ ${ }^{a}$ CNR-IBIM, Clinical Epidemiology and Physiopathology of Renal Diseases and Hypertension of Reggio Calabria, \\ Reggio Calabria, Italy; ${ }^{b}$ ERA-EDTA Registry, Department of Medical Informatics, Academic Medical Center, \\ University of Amsterdam, Amsterdam, and ${ }^{\mathrm{C}}$ Department of Clinical Epidemiology, Leiden University Medical \\ Center, Leiden, The Netherlands
}

\section{Key Words}

Confounding • Mantel-Haenszel formula $\cdot$ Odds ratio •

Relative risk $\cdot$ Stratification

\begin{abstract}
Stratification allows to control for confounding by creating two or more categories or subgroups in which the confounding variable either does not vary or does not vary very much. The Mantel-Haenszel formula is applied in cohort and in casecontrol studies to calculate an overall, unconfounded, effect estimate of a given exposure for a specific outcome by combining stratum-specific relative risks (RR) or odds ratios (OR). Stratum-specific RRs or ORs are calculated within each stratum of the confounding variable and compared with the corresponding effect estimates in the whole group (that is, with the unstratified RR or OR). The use of the Mantel-Haenszel formula presents some limitations: (1) if there is more than a single confounder, the application of this formula is laborious and demands a relatively large sample size, and (2) this method requires continuous confounders to be constrained into a limited number of categories thus potentially generating residual confounding (a phenomenon particularly relevant when the variable is categorized into few strata). In the stratified analysis, residual confounding can be minimized by increasing the number of strata, a possibility strictly dependent on sample size.

Copyright $\odot 2010$ S. Karger AG, Basel
\end{abstract}

\section{Introduction}

Confounding occurs in etiological research when the relationship between a given exposure and a specific disease/outcome is distorted (confused) by the influence of a third variable or group of variables (confounders) [1]. In controlling for confounding a researcher aims to obtain an unbiased estimate of the causal relationship between exposure and outcome. Confounding can be dealt with in the study design phase (by randomization, restriction and matching) and/or during data analysis (by stratification and multivariate analysis). Here we focus on stratification, a statistical technique that allows to control for confounding by creating two or more categories (strata) in which the confounding variable either does not vary or does not vary very much. There are two methods to adjust for confounding by stratification: pooling analysis by the Mantel-Haenszel formula [2] and standardization [3]. In this paper we focus on the Mantel-Haenszel method and in the next one on direct and indirect standardization.

\section{Stratification}

Stratification is the simplest method to control confounding during data analysis and represents the preliminary step for applying the Mantel-Haenszel formula and

\section{KARGER}

Fax +41613061234 E-Mail karger@karger.ch www.karger.com
Dr. Giovanni Tripepi, MSc

CNR-IBIM, Istituto di Biomedicina, Epidemiologia Clinica e Fisiopatologia delle Malattie Renali e dell'Ipertensione Arteriosa, c/o Euroline di Ascrizzi Vincenzo Via Vallone Petrara No. 55/57, IT-89124 Reggio Calabria (Italy)

Tel. +39 0965397 010, Fax +39 0965 26879, E-Mail gtripepi@ibim.cnr.it 


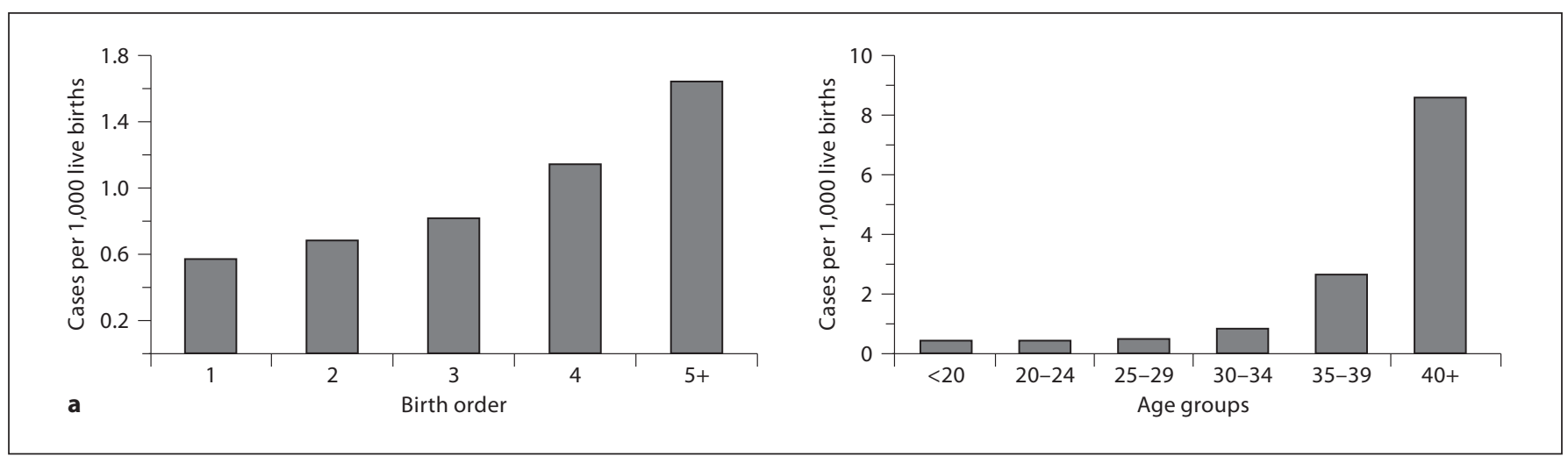

Fig. 1. a Association of the frequency of Down syndrome (cases per 1,000 live births) with birth order and maternal age. b Analysis of Down syndrome cases stratified by birth order and maternal age [adapted from 4]. See text for more details.

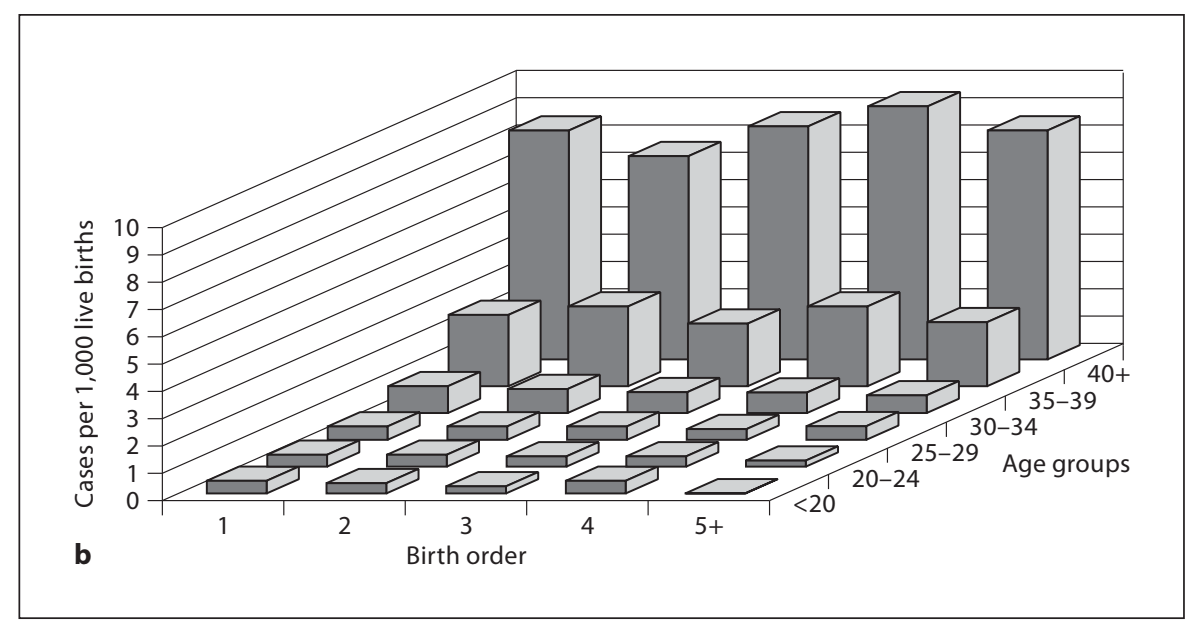

standardization. This technique was applied to investigate whether the birth order is causally implicated in the high frequency of Down syndrome independently of the confounding effect of the maternal age [4]. A crude analysis showed that the frequency of Down syndrome increases in close parallelism with birth order (fig. 1a) indicating that high birth order may be a risk factor for Down syndrome. The maternal age is strongly and directly associated with birth order and strictly related to the frequency of Down syndrome (fig. 1a). To ascertain whether maternal age is a confounder for the birth order-Down syndrome link, a stratified analysis was performed. As shown in figure $1 b$, in each birth order category the frequency of Down syndrome increased according to the maternal age while in each age category the birth order did not affect the frequency of Down syndrome. Thus, the crude association between birth order and Down syndrome is the mere result of the confounding effect of maternal age.

\section{Mantel-Haenszel Formula}

The Mantel-Haenszel formula allows to calculate an overall, unconfounded, that is adjusted, effect estimate of a given exposure for a specific disease/outcome by combining (pooling) stratum-specific relative risks (RR) or odds ratios (OR). Stratum-specific RRs or ORs are calculated within each stratum of the confounding variable and compared with the corresponding effect estimates in the whole group (that is, with unstratified RR or OR). When the effect estimates are roughly homogenous across strata and do not differ from that in the whole group, there is no confounding. Vice versa, when the effect estimates are substantially similar across strata but differ from that in the whole group, confounding is present [5]. When comparing stratified and unstratified effect estimates, epidemiologists consider as relevant a RR or OR difference by more than $10 \%$ [5]. When the magnitude of effect estimates varies by the levels of a third variable, e.g. individuals have or do not have cardiovascular disease (i.e., there is heterogeneity across strata and we are inter- 
Table 1. Number of deaths/survivors in the study population according to the presence/absence of $C$. pneumoniae infection [modified after 6]

\begin{tabular}{lll}
\hline C. pneumoniae infection & Deaths & Survivors \\
\hline Seropositive $(\mathrm{n}=89)$ & 38 & 51 \\
Seronegative $(\mathrm{n}=138)$ & 40 & 98 \\
\hline
\end{tabular}

ested to deal with it), the Mantel-Haenszel formula is not applicable because this circumstance reflects effect modification, an issue that will be discussed in a future article of this series.

There are five steps for assessing confounding through the Mantel-Haenszel formula: (1) calculate the crude RR or OR (i.e. without stratifying); (2) stratify by the confounding variable and calculate stratum-specific RR or OR; (3) assess the homogeneity of the effect estimates across strata and compare stratified and unstratified RRs or ORs; (4) if there is homogeneity in effect estimates across strata then calculate the overall, adjusted RR or OR by the Mantel-Haenszel formula, and (5) if there is heterogeneity and we are interested in effect modification, stratum-specific effect estimates should be reported separately.

\section{Example 1: The Mantel-Haenszel Formula in Follow-Up Studies}

Zoccali et al. [6] investigated the association between Chlamydia pneumoniae infection and mortality in a cohort of 227 end-stage renal disease (ESRD) patients followed up for 40 months. Patients were divided into two groups according to the presence/absence of antibodies for C. pneumoniae: 138 patients were classified as seronegative and the remaining 89 patients as seropositive (table 1).

During the follow-up, the proportion of deaths was significantly higher in seropositive than in seronegative patients (43 vs. $29 \%, \mathrm{p}<0.05$ ) (table 1). The RR of death (i.e. the ratio between the proportion of deaths in seropositive and in seronegative patients) was 1.47 (95\% confidence interval (CI): 1.03-2.10) indicating that patients with $C$. pneumoniae infection had a significantly higher risk of mortality when compared to those without (excess risk: $+47 \%$ ). If C. pneumoniae infection is causally implicated in the high risk of death in ESRD patients, the relationship between Chlamydia infection and mortality should be independent of other risk factors. In this study, age appeared to be a potential confounder [1] for the interpretation of the Chlamydia infection-mortality link because it was related to both Chlamydia infection (expo- sure) and mortality (outcome). Furthermore, there is no scientific evidence that age is an intermediate factor mediating this link. To assess whether the association between Chlamydia infection and death was independent of age, a stratified analysis was performed by dividing the study population into two groups (below/above the median value of age in the study population, 62 years) and in each age stratum the relationship between Chlamydia infection and death was computed. In table 2, ESRD patients who died and those who survived are rearranged according to both Chlamydia infection (the exposure) and age categories (the potential confounder).

Analysis of data stratified by age shows that: (1) there is no significant excess risk of death associated to Chlamydia infection in both age categories (95\% CIs include $1)$; (2) the RR of death was homogenous across strata $(\mathrm{RR}=1.08$ for patients aged $<62$ years and $\mathrm{RR}=1.28$ for those aged $\geq 62$ years), and (3) unstratified and stratified effect estimates differ by more than $10 \%$ (table 2 ). These results clearly indicate that the apparent strong link between Chlamydia infection and death risk that emerged in the unstratified analysis ( $\mathrm{RR}=1.47,95 \% \mathrm{CI}: 1.03-2.10)$ was due to the confounding effect of age. To calculate the overall RR of death associated to C. pneumoniae infection adjusting for the confounding effect of age, stratum-specific RRs are pooled by the Mantel-Haenszel formula.

The preliminary step of applying the Mantel-Haenszel formula to a follow-up study is to construct a $2 \times 2$ table in which the outcome occurrence is reported in columns and the distribution of exposure in rows (see below). The $2 \times 2$ table is then subdivided into smaller $2 \times 2$ tables according to levels of the confounder the investigator decides to test (see table 2). In general terms we have:

$\begin{array}{llll}\text { Exposure } & \text { Yes } & \text { No } & \\ \text { Yes } & \mathrm{a}_{\mathrm{i}} & \mathrm{b}_{\mathrm{i}} & \mathrm{N}_{1 \mathrm{i}} \\ \text { No } & \mathrm{c}_{\mathrm{i}} & \mathrm{d}_{\mathrm{i}} & \mathrm{N}_{0 \mathrm{i}} \\ & \mathrm{m}_{\mathrm{li}} & \mathrm{m}_{0 \mathrm{i}} & \mathrm{n}_{\mathrm{i}} \\ & \sum_{\mathrm{i}=1}^{s} \frac{\mathrm{a}_{\mathrm{i}} \mathrm{n}_{0 \mathrm{i}}}{\mathrm{n}_{\mathrm{i}}} & \\ \mathrm{RR}_{\text {Mantel-Haenszel }}=\frac{\mathrm{c}_{\mathrm{i}} \mathrm{n}_{1 \mathrm{i}}}{\mathrm{n}_{\mathrm{i}}} & \end{array}$

for strata i: from 1 to $s$.

In our instance:

$$
\mathrm{RR}_{\text {Mantel-Haenszel }}=\frac{\frac{7 \times 84}{116}+\frac{31 \times 54}{111}}{\frac{17 \times 32}{116}+\frac{23 \times 57}{111}}=1.22(95 \% \mathrm{CI}: 0.78-1.92)
$$

Nephron Clin Pract 2010;116:c317-c321 
Table 2. Number of deaths/survivors in the study population according to both C. pneumoniae infection and age categories [modified after 6]

\begin{tabular}{|c|c|c|c|c|c|c|}
\hline & \multicolumn{3}{|c|}{ Age $<62$ years $(n=116)$} & \multicolumn{3}{|c|}{ Age $\geq 62$ years $(n=111)$} \\
\hline & deaths & survivors & total & deaths & survivors & total \\
\hline \multicolumn{7}{|c|}{ C. pneumoniae infection } \\
\hline Seropositive & 7 & 25 & 32 & 31 & 26 & 57 \\
\hline Seronegative & 17 & 67 & 84 & 23 & 31 & 54 \\
\hline Total & 24 & 92 & 116 & 54 & 57 & 111 \\
\hline Stratum-specific & \multicolumn{3}{|c|}{$\begin{array}{l}\mathrm{RR}=1.08 \\
(95 \% \text { CI: } 0.50-2.36, \mathrm{p}=\mathrm{NS})\end{array}$} & \multicolumn{3}{|c|}{$\begin{array}{l}\mathrm{RR}=1.28 \\
(95 \% \mathrm{CI}: 0.86-1.89, \mathrm{p}=\mathrm{NS})\end{array}$} \\
\hline
\end{tabular}

Table 3. Distribution of lung cancer cases in alcohol abusers and non-abusers

\begin{tabular}{lll}
\hline Alcohol abuse & Cases (lung cancer) & Controls \\
\hline Yes & 88 & 68 \\
No & 62 & 82 \\
\hline
\end{tabular}

The $95 \% \mathrm{CI}$ of the $\mathrm{RR}_{\mathrm{MH}}$ is calculated by using a standard formula [4].

The authors concluded that after data adjustment for the confounding effect of age, the RR of death did not differ in patients with and without $C$. pneumoniae infection (the 95\% CI includes 1). Age engendered 'positive confounding' because it determined an overestimation of death risk associated with Chlamydia infection.

\section{Example 2: The Mantel-Haenszel Formula in \\ Case-Control Studies}

Here we consider a hypothetical case-control study investigating the association between alcohol abuse and lung cancer in a sample of 300 individuals including 150 lung cancer cases and 150 healthy subjects (table 3 ).

The OR of lung cancer (i.e. the ratio between the odds of lung cancer in alcohol abusers $(88 / 68=1.29)$ and the odds of the same disease in non-abusers $(62 / 82=0.76))$ was 1.70 (95\% CI: 1.08-2.70) indicating that the odds of lung cancer was significantly higher in alcohol abusers than in non-abusers. If alcohol abuse is implicated in the pathogenesis of lung cancer, the relationship between alcohol abuse and lung cancer should be independent of other risk factors. In this study, smoking is a potential confounder because it is associated with both alcohol in- take and lung cancer and it is not in the potential causal pathway mediating this link. The confounding effect of smoking on the alcohol intake-lung cancer link can be removed by the Mantel-Haenszel formula (table 4).

Data analysis stratified by smoking showed that: (1) there was no significant excess probability of lung cancer associated to alcohol abuse both in smokers and in nonsmokers (95\% CIs include 1); (2) the OR of lung cancer was homogenous across strata $(\mathrm{OR}=1.06$ in non-smokers and $\mathrm{OR}=1.0$ in smokers), and (3) unstratified and stratified ORs differ by more than $10 \%$ (table 4). These results indicate that the apparent strong link between alcohol abuse and lung cancer which emerged in the unstratified analysis (OR $=1.70,95 \% \mathrm{CI}: 1.08-2.70)$ was due to the confounding effect of smoking. To calculate the overall OR of lung cancer associated to alcohol abuse adjusting for the confounding effect of smoking, stratum-specific ORs are pooled by the Mantel-Haenszel formula. Since here we deal with a case-control study rather than with a follow-up study, the $2 \times 2$ table was constructed by reporting the exposure distribution in columns and the outcome in rows (see below).

\section{Exposure}

$$
\begin{array}{llll}
\text { Outcome } & \text { Yes } & \text { No } & \\
\text { Yes } & \mathrm{a}_{\mathrm{i}} & \mathrm{b}_{\mathrm{i}} & \mathrm{n}_{1 \mathrm{i}} \\
\text { No } & \mathrm{c}_{\mathrm{i}} & \mathrm{d}_{\mathrm{i}} & \mathrm{n}_{0 \mathrm{i}} \\
& \mathrm{m}_{1 \mathrm{i}} & \mathrm{m}_{0 \mathrm{i}} & \mathrm{n}_{\mathrm{i}} \\
& \\
\text { OR }_{\text {Mantel-Haenszel }}=\frac{\sum_{\mathrm{i}=1}^{\mathrm{s}} \frac{\mathrm{a}_{\mathrm{i}} \mathrm{d}_{\mathrm{i}}}{\mathrm{n}_{\mathrm{i}}}}{\sum_{\mathrm{i}=1}^{\mathrm{b}} \frac{\mathrm{b}_{\mathrm{i}} \mathrm{c}_{\mathrm{i}}}{\mathrm{n}_{\mathrm{i}}}}
\end{array}
$$

for strata i: from 1 to $s$. 
Table 4. Distribution of lung cancer cases in alcohol abusers and non-abusers stratifying by smoking

\begin{tabular}{|c|c|c|c|c|c|c|}
\hline & \multicolumn{3}{|c|}{ Non-smokers $(\mathrm{n}=81)$} & \multicolumn{3}{|c|}{ Smokers $(\mathrm{n}=219)$} \\
\hline & $\begin{array}{l}\text { with lung } \\
\text { cancer }\end{array}$ & $\begin{array}{l}\text { no lung } \\
\text { cancer }\end{array}$ & total & $\begin{array}{l}\text { with lung } \\
\text { cancer }\end{array}$ & $\begin{array}{l}\text { no lung } \\
\text { cancer }\end{array}$ & total \\
\hline \multicolumn{7}{|l|}{ Alcohol abuse } \\
\hline Yes & 53 & 15 & 68 & 35 & 53 & 88 \\
\hline No & 10 & 3 & 13 & 52 & 79 & 131 \\
\hline Total & 63 & 18 & 81 & 87 & 132 & 219 \\
\hline Stratum-specific & \multicolumn{3}{|c|}{$\begin{array}{l}\mathrm{OR}=1.06 \\
(95 \% \mathrm{CI}: 0.26-4.35, \mathrm{p}=\mathrm{NS})\end{array}$} & \multicolumn{3}{|c|}{$\begin{array}{l}\mathrm{OR}=1.00 \\
(95 \% \mathrm{CI}: 0.58-1.74, \mathrm{p}=\mathrm{NS})\end{array}$} \\
\hline
\end{tabular}

In our instance:

$$
\mathrm{OR}_{\text {Mantel-Haenszel }}=\frac{\frac{53 \times 3}{81}+\frac{35 \times 79}{219}}{\frac{10 \times 15}{81}+\frac{52 \times 53}{219}}=1.01(95 \% \text { CI: } 0.60-1.69)
$$

The $95 \% \mathrm{CI}$ of the $\mathrm{OR}_{\mathrm{MH}}$ is calculated by using a standard formula [4].

After data adjustment for the confounding effect of smoking, the OR of lung cancer did not differ in alcohol abusers and non-abusers (the 95\% CI includes 1). Smoking engendered 'positive confounding' because it determined an overestimation of the risk associated with alcohol abuse.

There are two important limitations of controlling for confounding by the Mantel-Haenszel formula: (1) if there is more than a single confounder, the application of this formula is laborious because of the higher number of strata and demands a relatively large sample size, and (2) this method requires continuous confounders to be constrained into a limited number of categories thus potentially generating residual confounding (a phenomenon particularly relevant when the variable is categorized into few strata). In example 1 , to remove the confounding effect of age on the Chlamydia infection-mortality link, the study population was stratified in two age categories (below/above 62 years). However, this stratification did not guarantee that the confounding effect of age be completely removed. In fact, in the first category age ranged from 18 to 61 years and in the second category from 62 to 90 years. As a consequence, within each stratum a substantial residual confounding of age still remained. For this reason, Zoccali et al. [6] performed a multivariate analysis by considering age as a continuous variable. The application of multivariate techniques to control for con- founding will be discussed in a future article of this series. In general, in the context of stratification, residual confounding can be minimized by increasing the number of strata, a possibility strictly dependent on sample size.

\section{Conclusions}

The Mantel-Haenszel formula is a simple technique that can be applied for controlling for confounding. This method combines stratum-specific RRs or ORs. The pooling estimate provides an average of the stratum-specific RRs or ORs with weights proportional to the number of individuals in each stratum. This method is particularly well suited in clinical and epidemiological research to remove confounding in studies with relatively large sample size and with a relatively low number of potential confounders. An important limitation of this technique is residual confounding.

\section{References}

1 Jager KJ, Zoccali C, Macleod A, Dekker FW: Confounding: what it is and how to deal with it. Kidney Int 2008;73:256-260.

2 Mantel N, Haenszel W: Statistical aspects of the analysis of data from retrospective studies of disease. J Natl Cancer Inst 1959;22:719-748.

3 Szklo M, Nieto FJ: Epidemiology. Beyond the Basics. Boston, Jones \& Bartlett, 2004, pp 265-273.

4 Rothman KJ: Epidemiology. An Introduction. New York, Oxford University Press, 2002, pp 101-105, 144-158.

5 Maldonado G, Greenland S: Simulation study of confounder-selection strategies. Am J Epidemiol 1993;138:923-936.

6 Zoccali C, Mallamaci F, Tripepi G, Parlongo S, Cutrupi S, Benedetto FA, Bonanno G, Seminara G, Fatuzzo P, Rapisarda F, Malatino LS: Chlamydia pneumoniae, overall and cardiovascular mortality in endstage renal disease. Kidney Int 2003;64:579-584. 